\title{
Femoral Nerve Palsy with Patella Fracture
}

\author{
Sang Hyoung Lee, $\mathrm{MD}^{1}$, Tong Joo Lee, $\mathrm{MD}^{2}$, Min Su Woo, $\mathrm{MD}^{2}$, and Dae Gyu Kwon, $\mathrm{MD}^{2}$ \\ ${ }^{1}$ Department of Orthopaedic Surgery, Dongguk University Hospital, Ilsan; ${ }^{2}$ Department of Orthopaedic Surgery, Inha University Hospital, Incheon, Korea
}

Femoral neuropathy may be associated with various etiologies and can cause severe walking disability. We present the case of a 25 -year-old woman who underwent surgical repair for a patella fracture and complained of lower extremity pain, paresthesia, and weakness postoperatively. Electromyography and magnetic resonance imaging (MRI) revealed partial peripheral neuropathy of the left femoral nerve associated with the patella fracture. To our knowledge, this is the first reported case of femoral neuropathy associated with a patella fracture.

Keywords: Patella, Fracture, Femoral neuropathy, Muscle weakness

Femoral mononeuropathy is an unusual neuropathy that can cause severe walking disability ${ }^{1)}$. In general, femoral nerve injuries have a good prognosis and a tendency to recover over a period of days to months. Actual persistent palsies are rare in the literature ${ }^{2)}$. Therefore, the condition tends to be underdiagnosed because of the rapid recovery by the nerve.

Femoral neuropathy may be associated with trauma, diabetes, hypotension, drug abuse, neoplastic or paraproteinemic disease, lumbar spondylosis, renal implant surgery, acute hyperextension of the thigh, total hip arthroplasty, patellar dislocation, or abdominal hysterectomy. It has also been reported after the use of a pneumatic tourniquet. Furthermore, it has been suggested that prolonged placement in the lithotomy position may lead to neuropathy due to compression of the nerve by the inguinal ligament $^{3-5)}$.

There has been no previous report of femoral neuropathy involved with patella fracture. Here, we present for the first time a case of femoral neuropathy after patella fracture.

Received March 20, 2013; Revised (1st) June 3, 2013; (2nd) July 2, 2013; (3rd) July 31, 2013; Accepted August 5, 2013

Correspondence to: Dae Gyu Kwon, MD

Department of Orthopaedic Surgery, Inha University Hospital, 27

Inhang-ro, Jung-gu, Incheon 400-711, Korea

Tel: +82-32-890-3044, Fax: +82-32-890-3467

E-mail: gundegy77@gmail.com

This is an Open Access article distributed under the terms of the Creative Commons Attribution Non-Commercial License (http://creativecommons.org/licenses/by-nc/3.0/) which permits unrestricted non-commercial use, distribution, and reproduction in any medium, provided the original work is properly cited.

\section{Case Report}

A 25-year-old active young woman (weight $57 \mathrm{~kg}$, height 1.64 m) suffered a left patella fracture due to a slip and fall. She had a medical history of right knee arthroscopy in 2001 due to a cruciate ligament injury. Complete blood count, electrolyte panel, coagulation screening, electrocardiography, and plain radiographic findings were all within normal limits. A physical examination revealed moderate tenderness and swelling in the left thigh and knee area. We could not check motor grade of the left knee due to severe pain. Open reduction and internal fixation with a tension band wiring was performed under spinal anesthesia three days after admission to the emergency room. A pneumatic tourniquet was inflated to $370 \mathrm{mmHg}$ at the onset of the procedure and was deflated 80 minutes after surgery. Her leg was elevated to 30 degrees immediately after surgery, and an ice bag was applied to the operation site. Immediate full weight bearing and range of motion were allowed. There were no signs of infection. The patient was discharged with a crutch four days after the operation.

The patient returned the following week for consultation. She had marked pain in the left knee area, difficulty in extending the knee, and weakness of the quadriceps muscle. She was referred to a rehabilitation physician and a neurologist for electromyography-nerve conduction velocity (EMG-NCV) testing, but she refused the test.

Eight weeks after being discharged, she was readmitted because of persistent pain and neurologic symptoms. At that time, manual muscle testing of the left lower limb showed grade IV hip abduction, grade $\mathrm{V}$ adduction, grade III hip flexion, grade II 


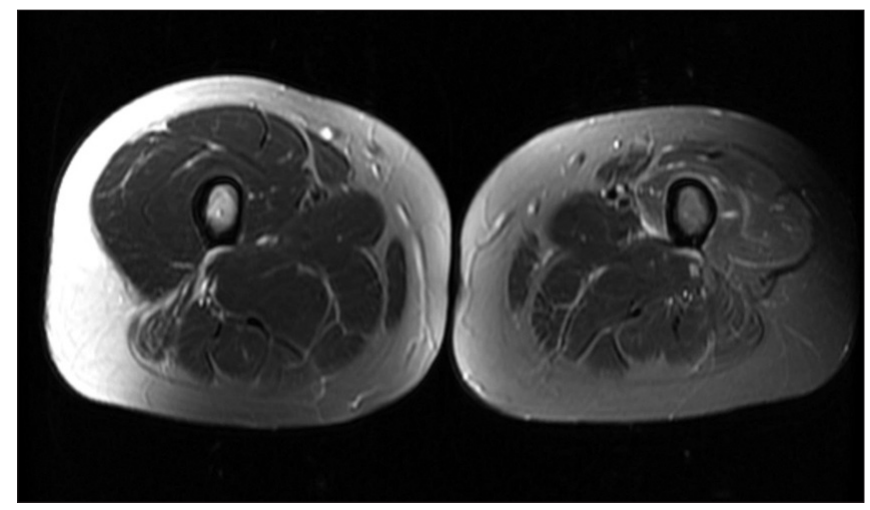

Fig. 1. Magnetic resonance imaging of the thigh shows diffuse T2 high signal intensity and decreased volume in the left quadriceps group, suggesting neurogenic atrophy.

knee extension, grade $\mathrm{V}$ ankle dorsiflexion, and grade $\mathrm{V}$ plantar flexion. Pinprick sensation of the femoral nerve and lateral cutaneous nerve in the thigh area was decreased to 8 points on a scale from 0 to 10 points. Reflexes at the left patella were not assessable. Knee pain was measured as 5 on a visual analogue scale. MRI of the knee showed no evidence of abnormalities of the anterior cruciate ligament, posterior cruciate ligament, lateral collateral ligament, medial collateral ligament, or meniscus abnormality, except for slight increase in the amount of joint effusion. However, evaluations of the patella, infrapatellar fat pad, quadriceps tendon, patellar tendon, and anterior horn of medial/lateral menisci were limited due to severe metallic artifacts. Diffuse T2 high signal intensity was observed in the anterior compartment of the left thigh, suggesting an acute denervation change related to left femoral neuropathy. Furthermore, the whole volume of the muscles of the left thigh was reduced, suggesting combined disuse atrophy (Fig. 1). MRI of the lumbar spine depicted a normal spinal cord, L4 nerve roots, and lumbar plexus, except for bulging discs with an annular tear at L3-4, L4-5, and L5-S1. In addition, mildly increased echogenicity was noted in the left anterior compartment muscle with decreased volume by joint ultrasonography, suggesting disuse atrophy or a denervation change.

An EMG study was performed on the left tibialis anterior, gastrocnemius medialis, vastus lateralis, vastus medialis, rectus femoris, adductor brevis, gluteus medius, and lumbar paraspinal (L3 and 4) muscles. Positive sharp waves and fibrillation potentials were observed in the vastus lateralis, vastus medialis, and rectus femoris muscles by needle electromyography. Furthermore, a sensory nerve conduction study (NCS) revealed reduced conduction for the left saphenous nerve. One week after the test, a follow-up EMG study was done by another neurologist, and the results were the same.
Accordingly, the study established the presence of partial peripheral neuropathy of the left femoral nerve. The patient was referred for electrical stimulation therapy and steroid therapy but refused any further treatments. The internal fixation device was removed at two months after surgery due to severe pain, and subsequently, the knee pain scale improved to visual analogue scale 3 five days after the device removal.

\section{Discussion}

Deficits of motor and sensory function resulting from femoral neural injury are probably underestimated because most cases are self-limited and thus underreported. Hence, the significance of this report is that it presents the first reported case of femoral neuropathy accompanied by patella fracture.

The femoral nerve has its origins in the posterior divisions of the L2, L3, and L4 branches of the lumbar plexus ${ }^{6}$. The main sensory branches of the femoral nerve are the anterior femoral cutaneous and saphenous nerves, which supply the anteromedial aspect of the thigh and medial side of the leg, respectively. The motor branches supply the sartorius, iliacus, pectineus, rectus femoris, vastus lateralis, vastus intermedius, and vastus medialis muscles $^{6,7)}$.

Innervation to the vastus lateralis muscle runs in a space restricted by the fascial surfaces of the vastus intermedius and rectus femoris muscles anteriorly and posteriorly and the edges of the vastus medialis and vastus lateralis muscles on each side. Consequently, neuropathy can occur due to entrapment of the nerve by the vastus lateralis muscle ${ }^{3)}$. Furthermore, femoral nerve injury can give rise to severe difficulty in ambulation, due to loss of innervation of the anterior thigh musculature, which is essential for walking ${ }^{7}$.

As mentioned above, femoral neuropathy may manifest in several ways. In our case, the patient had femoral neuropathy and atrophy of the left quadriceps muscle group accompanied by sensory changes in the distribution of the lateral cutaneus nerve of the thigh.

The initial differential diagnosis for this weakness included disuse atrophy, neuropraxia caused by a pneumatic tourniquet, femoral neuropathy, lumbar plexopathy, and radiculopathy at the L3 or L4 level. Other potential causes had been ruled out by blood test, medical history, clinical examination, EMG-NCV, joint ultrasonography, and MRI of the knee, thigh, and lumbar spine. The patient was allowed to walk and exercise to prevent disuse atrophy. Routine blood test results and vital signs were examined to rule out diabetes, hypotension, and drug abuse. Electrodiagnostic 
testing and MRI were used to localize the site of the pathology. Electrodiagnostic testing showed showed abnormalities in only the muscles innervated by the femoral nerve with sparing of the iliopsoas, suggesting an injury to the femoral nerve at or near the inguinal ligament. And we could rule out lumbar plexopathy and lumbar radiculopathy based on the MRI findings.

Stretch injuries of the femoral nerve following hip hyperextension have been reported in previous studies. Miller and Benedict ${ }^{8)}$ reported the case of a dancer who suffered femoral neuropathy following exercises involving extreme hip extension and knee flexion. Serial evaluation could not be performed in the case because of loss to follow-up. As determined by electrodiagnostic testing and MRI, stretch injury at or near the inguinal ligament, distal to the muscular branches of the iliopsoas and sensory branches of the lateral cutaneus nerve of thigh, was a probable cause $^{5}$. Therefore, it is conceivable that femoral neuropathy or neuropraxia in this case was caused by a stretch injury of the femoral nerve at the time of the slip and fall or during the operation.

\section{Conclusions}

Clinicians should have a high index of suspicion for neurological compromise following all types of fractures, and if needed, further evaluations should be conducted promptly. Surgeons should be aware of this potential morbidity and take care not to compress over the inguinal area during operation to eliminate the risk of this complication.

\section{Conflict of Interest}

No potential conflict of interest relevant to this article was reported.

\section{Acknowledgments}

This paper was supported by the research fund from Inha University (44827-01).

\section{References}

1. Tetsworth K, Paley D. Malalignment and degenerative arthropathy. Orthop Clin North Am. 1994;25:367-77.

2. Kornbluth ID, Freedman MK, Sher L, Frederick RW. Femoral, saphenous nerve palsy after tourniquet use: a case report. Arch Phys Med Rehabil. 2003;84:909-11.

3. Muellner T, Ganko A, Bugge W, Engebretsen L. Isolated femoral mononeuropathy in the athlete. Anatomic considerations and report of two cases. Am J Sports Med. 2001;29:814-7.

4. Ducic I, Dellon L, Larson EE. Treatment concepts for idiopathic and iatrogenic femoral nerve mononeuropathy. Ann Plast Surg. 2005;55:397-401.

5. Shin CS, Davis BA. Femoral neuropathy due to patellar dislocation in a theatrical and jazz dancer: a case report. Arch Phys Med Rehabil. 2005;86:1258-60.

6. Peirce C, O’Brien C, O'Herlihy C. Postpartum femoral neuropathy following spontaneous vaginal delivery. J Obstet Gynaecol. 2010;30:203-4.

7. Burke NG, Walsh M, O’Brien T, Synnott K. Diagnostic gait pattern of a patient with longstanding left femoral nerve palsy: a case report. J Orthop Surg (Hong Kong). 2010;18:3824.

8. Miller EH, Benedict FE. Stretch of the femoral nerve in a dancer: a case report. J Bone Joint Surg Am. 1985;67:315-7. 